\title{
Fibroblast growth factor receptor 1 promotes MG63 cell proliferation and is associated with increased expression of cyclin-dependent kinase 1 in osteosarcoma
}

\author{
WEI ZHOU ${ }^{1}$, YUE ZHU ${ }^{1}$, SONG CHEN ${ }^{1}$, RUIJUN XU ${ }^{1}$ and KUNZHENG WANG ${ }^{2}$ \\ ${ }^{1}$ Department of Orthopaedics, The Sixth People's Hospital Affiliated to Shanghai Jiaotong University, Shanghai 200233; \\ ${ }^{2}$ Department of Orthopaedics, The Second Hospital Affiliated to Xi'an Jiaotong University, Xi'an, Shaanxi 710004, P.R. China
}

Received November 12, 2014; Accepted August 5, 2015

DOI: $10.3892 / \mathrm{mmr} .2015 .4597$

\begin{abstract}
Osteosarcoma is the most common type of malignant bone tumor in adolescents and young adults. However, current understanding of osteosarcomagenesis remains limited. In the present study, the role of fibroblast growth factor receptor 1 (FGFR1) in human osteosarcoma cell proliferation was investigated, and the possible pathways that contribute to FGFR1-mediated osteosarcoma cell proliferation were examined using microarray analysis. The expression of FGFR1 in osteosarcoma tissues was assessed by reverse transcription-quantitative polymerase chain reaction and immunohistochemistry. The results demonstrated that FGFR1 was markedly increased in osteosarcoma tissues, and that the overexpression of FGFR1 in MG63 cells significantly promoted cell proliferation, as observed using the cell viability assay. In addition, FGFR1-mediated cell proliferation was closely associated with cell cycle re-distribution, as determined by microarray analysis. Western blotting identified that the expression of cyclin-dependent kinase 1 (CDK1) was correspondingly increased in response to the overexpression of FGFR1. These results indicated that FGFR1 contributes to cell proliferation in osteosarcoma MG63 cells, and FGFR1 mediated cell proliferation may be attributed to the regulation of the cell cycle regulator, CDK1. These findings provide evidence to support the potential use of molecule target therapy against FGFR1 as a promising strategy in osteosarcoma treatment and prevention.
\end{abstract}

\section{Introduction}

Osteosarcoma is the most common type of malignant bone tumor in adolescents and young adults. In $\sim 75 \%$ of cases,

Correspondence to: Dr Kunzheng Wang, Department of Orthopaedics, The Second Hospital Affiliated to Xi'an Jiaotong University, 157 Xiwu Road, Xi'an, Shaanxi 710004, P.R. China E-mail: wangkunzheng@163.com

Key words: fibroblast growth factor receptor 1, cyclin-dependent kinase 1, proliferation, MG63 cells, osteosarcoma patients suffering from osteosarcoma are aged between 15-25 years old, with a median onset age of 16 years old and a male predominance (1). Pain and swelling of the soft tissues are the most common symptoms in patients with osteosarcoma (2). Histologically, osteosarcoma is ascribed to the proliferation of malignant spindle cells and is characterized by osteoid, which is directly produced by sarcoma cells (3). However, although current understanding of the histological and clinical manifestations of osteosarcoma is increasing, knowledge regarding the onset of osteosarcoma remains limited.

Previous reports identifying fibroblast growth factor receptors (FGFRs) have significantly improved current understanding of human tumorigenesis (4-6). FGFRs are transmembrane tyrosine kinase receptors, which belong to the immunoglobulin (Ig) superfamily (7). FGFRs are known to be comprised of four members in humans; FGFR1, FGFR2, FGFR3 and FGFR4 (7). Structurally, the prototypical FGFR monomer consists of three domains: An extracellular domain, which mediates FGF binding; a transmembrane domain; and an intracellular tyrosine kinase domain (7). The binding of FGFs ligands to FGFRs directly induces receptor dimerization and finally activates FGFRs kinase activities, leading to initiation of the intracellular signaling network (7). Increasing evidence indicates that alteration of the FGF-FGFR signaling cascade may lead to cancer and is involved in organ development, tumor cell proliferation and metastasis (6,8-11). At present, three alterations have been identified as the predominant mechanisms that contribute to FGFR-mediated human tumorigenesis, including chromosomal translocations (12-14), receptor gene amplification (15-17) and FGFR-activating mutations $(18,19)$.

FGFR1, the first member of the FGFR family, has been investigated predominantly in the process of human tumorigenesis. Of note, FGFR1 overexpression is common in multiple types of tumor. A previous study demonstrated that, in breast cancer, FGFR1 amplification was one of the most common changes and accounted for $10 \%$ of breast cancer cases (20). Evidence has also revealed that the upregulation of FGFR1 increases cell proliferative ability, whereas its downregulation stimulates apoptosis in breast cancer (21). In addition, a previous study reported the existence of focal amplification 
of FGFR1 in non-small cell lung cancer and in $21 \%$ of lung adenocarcinoma cases (22). Furthermore, the number of FGFR1 copies has been identified as an independent prognostic factor in non-small cell lung cancer (23), and the FGFR inhibitor, ponatinib, can suppress the growth of non-small cell lung cancer cells exhibiting a high expression level of FGFR1 (24). FGFR1 has also found to be upregulated in prostate cancer (25), pancreatic ductal adenocarcinoma (26), oral squamous cell carcinoma (27), bladder cancer (28), ovarian cancer (29) and sarcoma (30). Although high expression levels of FGFR1 have been observed in a broad spectrum of types of cancer, its role in human bone diseases remains to be elucidated. To the best of our knowledge, FGFR1 has only been reported to be associated with fracture non-union (31).

The present study aimed to investigate the expression profile of FGFR1 in osteosarcoma and determine the possible mechanisms underlying FGFR-mediated osteosarcoma development, using high-throughput tissue microarray analysis Furthermore, the role of FGFR1 in osteosarcoma MG63 cell proliferation was examined.

\section{Materials and methods}

Reagents. FGFR1 cDNA was amplified from the human genome by polymerase chain reaction (PCR) and the amplified fragments were digested with HindIII and XhoI (Takara Biotechnology Co., Ltd., Dalian, China) and were inserted into the HindIII and XhoI sites of the pcDNA3.1-Flag vector (Invitrogen Life Technologies, Carlsbad, CA, USA), according to the manufacturer's instructions. Sequences were verified using DNA electrophoresis and sequencing. Dulbecco's modified Eagle's medium (DMEM; Corning Incorporated, New York, NY, USA), dimethylsulfoxide (DMSO; Sigma-Aldrich, St. Louis, MO, USA) and fetal bovine serum (FBS; Thermo Fisher Scientific, Waltham, MA, USA) were all purchased from commercial sources, as described. The anti-FGFR1 and actin antibodies were purchased from Santa Cruz Biotechnology, Inc. (Santa Cruz, CA, USA). Primary antibodies against CDK1 and CDK2 were obtained from Abcam (Cambridge, MA, USA).

Cell line and transfection. The MG63 human osteosarcoma cell line was obtained from Shanghai Institute of Biological Sciences, Chinese Academy of Sciences (Shanghai, China). The cells were cultured in DMEM with $10 \% \mathrm{FBS}$ at $37^{\circ} \mathrm{C}$ in a $5 \% \mathrm{CO}_{2}$ humidity-controlled incubator. The medium was refreshed every 2 days. The Flag-FGFR1 plasmid, which was constructed using the pcDNA3.1-basic vector, was transfected into the MG63 cells ( $1 \times 10^{6}$ cells $/ 6 \mathrm{~cm}$ dish) prior to cell confluence reaching 80\%. Lipofectamine 2000 (Invitrogen Life Technologies) was used as the transfection regent. Transfection efficiency was verified using a fluorescence microscope (DMI3000 B; Leica Microsystems GmbH, Wetzlar, Germany) and subsequent western blotting.

Patient samples and immunohistochemisty (IHC). A total of 65 patients who underwent surgery at The Sixth People's Hospital Affiliated to Shanghai Jiaotong University (Shanghai, China) between 2012 and 2014 were chosen subsequent to histological verification of osteosarcoma. None of the 65 patients received preoperative chemotherapy or radiotherapy. The examined population consisted of 65 patients (41 men and 24 women) with a median age of diagnosis of 51 years old (range, 33-75 years). The tissue, which was separate from the edge of tumor foci and was histologically judged to be free from adenocarcinoma cells, was used as a normal tissue control. All tissue samples were fixed in $4 \%$ formalin immediately subsequent to removal and were embedded in paraffin for immunohistochemical staining. The study was approved by the Ethics Committee of Shanghai Jiaotong University, and all patients provided informed consent.

IHC analysis was performed using osteosarcoma tissues and paired normal bone tissues. Paraffin-embedded tissues were first cut into $4 \mu \mathrm{m}$-thick sections, followed by deparaffinization and rehydration with xylene and ethanol. The sections were then subjected to antigen retrieval in $0.1 \mathrm{M}$ citrate acid buffer (Sangon Biotech Co., Ltd., Shanghai, China). Following washing in phosphate-buffered saline (PBS) three times, the sections were incubated with primary antibodies overnight at $4^{\circ} \mathrm{C}$. Following incubation, the sections were incubated with horseradish-peroxidase-labeled secondary antibody for $1 \mathrm{~h}$ at room temperature. Following washing in PBS three times, the sections were visualized using diaminobenzidine and images were captured (DM13000 B; Leica Microsystems GmbH).

Reverse transcription-quantitative PCR (RT-qPCR). Total RNA were isolated from samples from 65 patients using TRIzol reagent (Sigma-Aldrich). The cDNA (500 ng) was then prepared using an AMV Reverse Transcriptase synthesis kit (Promega Corporation, Madison, WI, USA), according to the manufacturer's instructions. qPCR was performed using a QuantiTect SYBR Green PCR kit (Qiagen, Shanghai, China). The qPCR cycle conditions were as follows: $95^{\circ} \mathrm{C}$ for $30 \mathrm{sec} 38$ cycles of $95^{\circ} \mathrm{C}$ for $5 \mathrm{sec}$ and $60^{\circ} \mathrm{C}$ for $34 \mathrm{sec}$. The amplification specificity was evaluated by melting curve analysis. The relative mRNA levels were determined using the $2^{-\Delta \mathrm{CT}}$ (CT; cycle threshold) formula, where $\Delta \mathrm{CT}=\mathrm{CT}$ (target gene) - CT (actin). All assays were performed in triplicate. $\beta$-actin was selected as the internal control. The following primers were used: Forward 5'-CTAAGATCCCGTCCATCGCC-3' and reverse 5'-GGAGCCCAGCACTTTGATCT-3'.

Western blot analysis. Following culture for 24 h, the MG63 cells were lysed using radioimmunoprecipitation assay buffer (Sigma-Aldrich), containing $150 \mathrm{mM} \mathrm{NaCl}, 1 \%$ sodium deoxycholate, $0.1 \%$ SDS, $1 \%$ Triton $\mathrm{X}-100,50 \mathrm{mM}$ Tris- $\mathrm{HCl}$ (pH 7.5) and $2 \mathrm{mM}$ EDTA), on ice for $20 \mathrm{~min}$. Following centrifugation at $4^{\circ} \mathrm{C}$ for $20 \mathrm{~min}(12,000 \mathrm{x} \mathrm{g})$, the supernatant was collected. Protein concentration was determined using a Bicinchoninic Acid Assay kit (Pierce Biotechnology, Rockford, IL, USA). Equal quantities of protein extract $(40 \mu \mathrm{g})$ were loaded into each lane of a 12\% SDS-PAGE gel (Sangon Biotech Co., Ltd.) for separation, and transferred onto polyvinylidene difluoride membranes (Bio-Rad Laboratories, Inc., Hercules, CA, USA). Following antigen blocking in Tris-buffered saline with $0.1 \%$ Tween 20 , supplemented with $5 \%$ skim milk for $1 \mathrm{~h}$, the membranes were incubated with the following primary antibodies overnight at $4^{\circ} \mathrm{C}$ : Monoclonal rabbit anti-EGFR1 (1:1,000; cat. no. ab32152; Epitomics, Inc., Burlingame, CA, USA) and monoclonal rabbit anti-CDK1 (1:1,000; cat. 
A

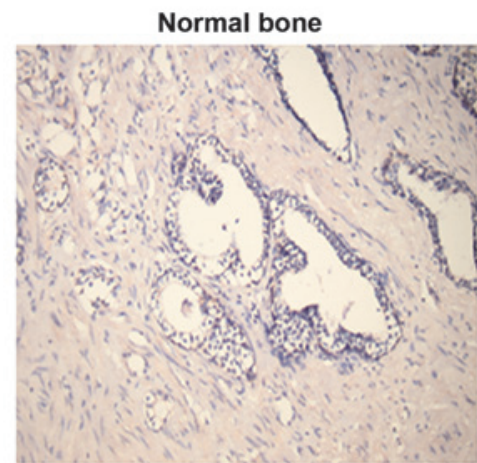

Osteosarcoma

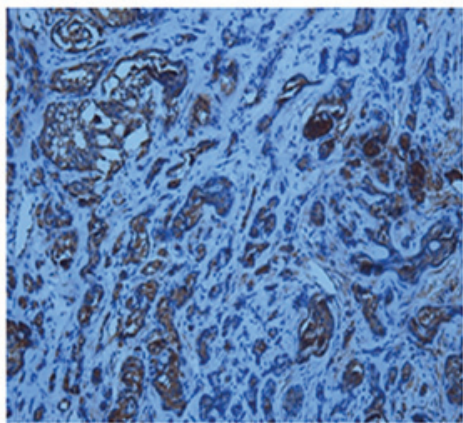

B

FGFR1 mRNA expression

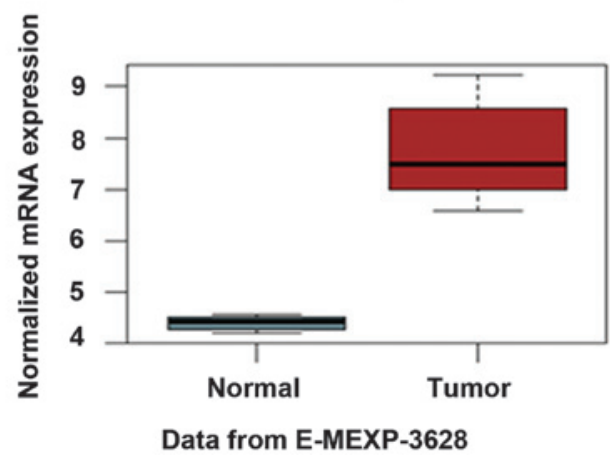

Figure 1. Expression of FGFR1 is high in osteosarcoma tissues. (A) IHC analysis of osteosarcoma and paired normal bone tissues with the FGFR1 antibody. The protein level of FGFR1 was significantly elevated in the osteosarcoma tissues (magnification, x200). (B) Determination of the mRNA level of FGFR1 in osteosarcoma and paired normal tissues. The changes in the mRNA expression levels were consistent with the results of the IHC analysis. Data are expressed as the mean \pm standard deviation. FGFR1, fibroblast growth factor receptor 1; ICH, immunohistochemisty.

no. 5524; Cell Signaling Technology, Inc., Danvers, MA, USA). Subsequently, the membranes were incubated with the goat anti-rabbit IgG (1:5,000; Santa Cruz Biotechnology, Inc.) secondary antibodies for $1 \mathrm{~h}$ at room temperature and were processed for electrochemiluminescent detection using an ECL system (Pierce Biotechnology).

Microarray analysis. To investigate the possible pathways contributing to the onset of FGFR1-mediated osteosarcoma, the present study performed microarray analysis in osteosarcoma samples. The focus of this investigation was predominantly on cell cycle changes, and bone remodeling changes were selected as a control. Data from the scanned arrays were extracted using Genome Studios software (v 1.6; Illumina, San Diego, CA, USA). Data processing and statistical analysis were performed using MATLAB 2011a (MathWorks, Natick, MA, USA), which was also used for multidimensional scaling analysis of the detected probes. For gene analysis, the microarray data were analyzed using the filtering criteria of adjusted $\mathrm{P}<0.05$ and $>15 \%$ change. To reveal genes with significant changes in expression, heatmap analysis was performed for visualization using MATLAB.

Cell viability assay. A Cell Counting Kit-8 (Dojindo Molecular Technologies, Inc., Kumamoto, Japan) was used to measure cell viability following FGFR1 transfection. The MG63 cells $\left(10^{3}\right)$ were seeded into a 96-well plate and incubated for $48 \mathrm{~h}$ at $37^{\circ} \mathrm{C}$ in $5 \% \mathrm{CO}_{2}$. At $48 \mathrm{~h}$ post-transfection, $10 \mu \mathrm{l}$ CCK-8 solution was added to each well and the cells were incubated for a further $4 \mathrm{~h}$ at $37^{\circ} \mathrm{C}$, following which the absorbance at $450 \mathrm{~nm}$ was measured using a microplate reader (Multiskan Spectrum; Thermo Fisher Scientific). Each experiment was repeated in triplicate, and observation of the cells was continued for 4 days to determine changes.

Statistical analysis. Data are representative of at least three independent replicate experiments performed in triplicate, and are expressed as the mean \pm standard deviation. Student's t-test was used to analyze the difference between two groups. $\mathrm{P}<0.05$ was considered to indicate a statistically significant difference. All statistical analyses were performed with SPSS statistical software, version 20.0 (IBM SPSS, Armonk, NY, USA).

\section{Results}

FGFRl is expressed at high levels in osteosarcoma tissues. To determine the expression level of FGFR1 in osteosarcoma tissues, the present study performed IHC analysis of osteosarcoma tissues and paired normal bone tissues using the FGFR1 antibody. The results demonstrated that the protein level of FGFR1 in the osteosarcoma tissues was notably higher than that in the paired normal bone tissues (Fig. 1A). The staining of FGFR1 was predominantly located in the plasma (Fig. 1A). In addition, the mRNA level of FGFR1 was determined in the two tissue groups. Consistently, FGFR1 exhibited a relative mRNA level of $\sim 4.5$ in the normal bone, whereas the relative mRNA level reached almost 7.5 in the osteosarcoma tissues 
A a

$\begin{array}{ll}\text { Dataset } & \text { E-MEXP-3628.FGFR1 } \\ \text { Phenotype } & \text { FGFR1 } \\ \text { Upregulated in class } & \text { FGFR1_neg } \\ \text { GeneSet } & \text { CELL_CYCLE_PHASE } \\ \text { Enrichment Score (ES) } & -0.27569094 \\ \text { Normalized Enrichment } & -1.4721138 \\ \text { Score (NES) } & \\ \text { Nominal p-value } & 0.0031347962 \\ \text { FDR q-value } & 0.11564929 \\ \text { FWER p-Value } & 1.0\end{array}$

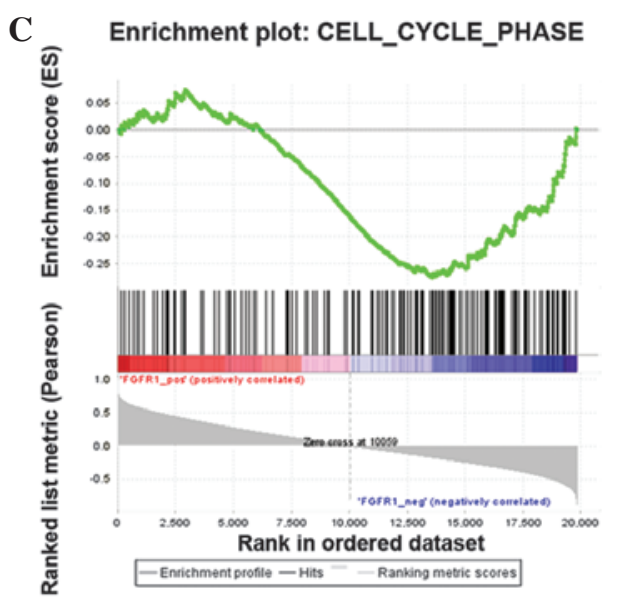

D

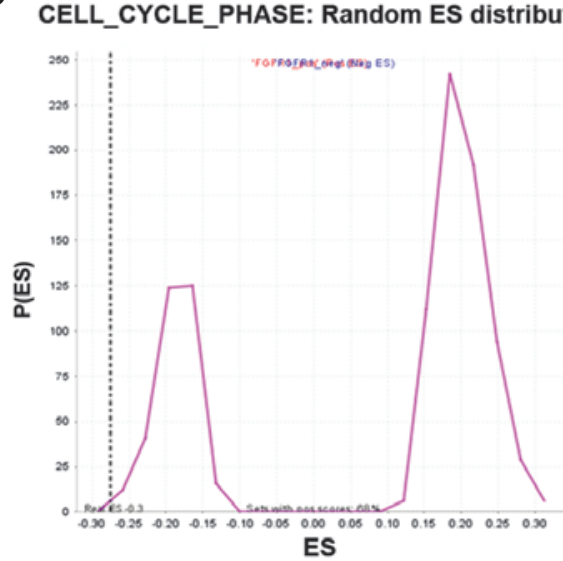

b

$\begin{array}{ll}\text { Dataset } & \text { E-MEXP-3628.FGFR1 } \\ \text { Phenotype } & \text { FGFR1 } \\ \text { Upregulated in class } & \text { FGFR1_pos } \\ \text { GeneSet } & \text { BONE_REMODELING } \\ \text { Enrichment Score (ES) } & 0.29843432 \\ \begin{array}{l}\text { Normalized Enrichment } \\ \text { Score (NES) }\end{array} & 1.0676486 \\ \text { Nominal p-value } & 0.36998254 \\ \text { FDR q-value } & 0.5754387 \\ \text { FWER p-Value } & 1.0\end{array}$

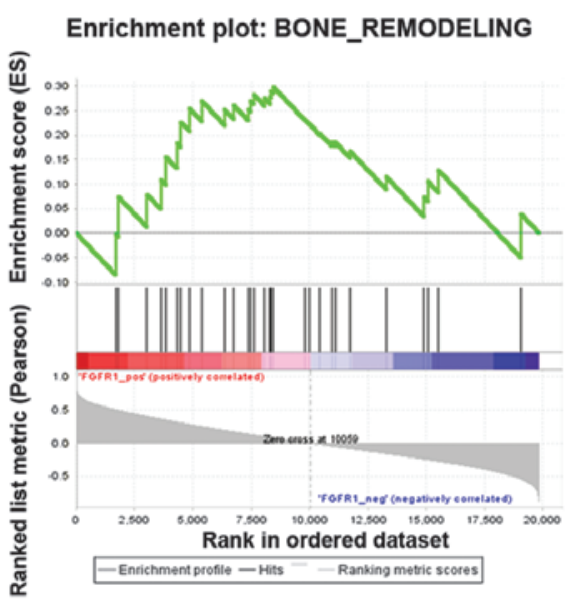

BONE_REMODELING: Random ES distribution

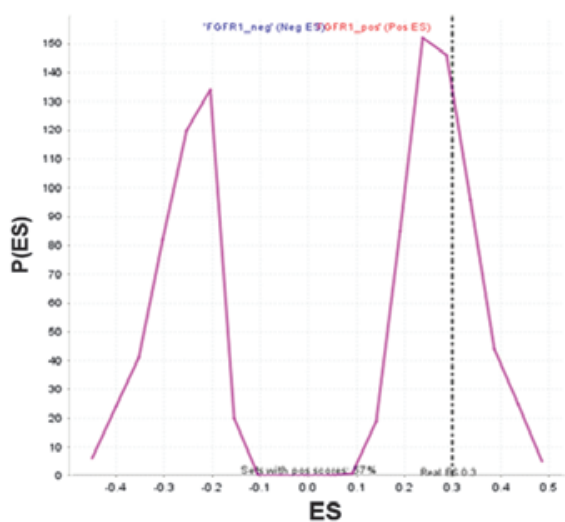

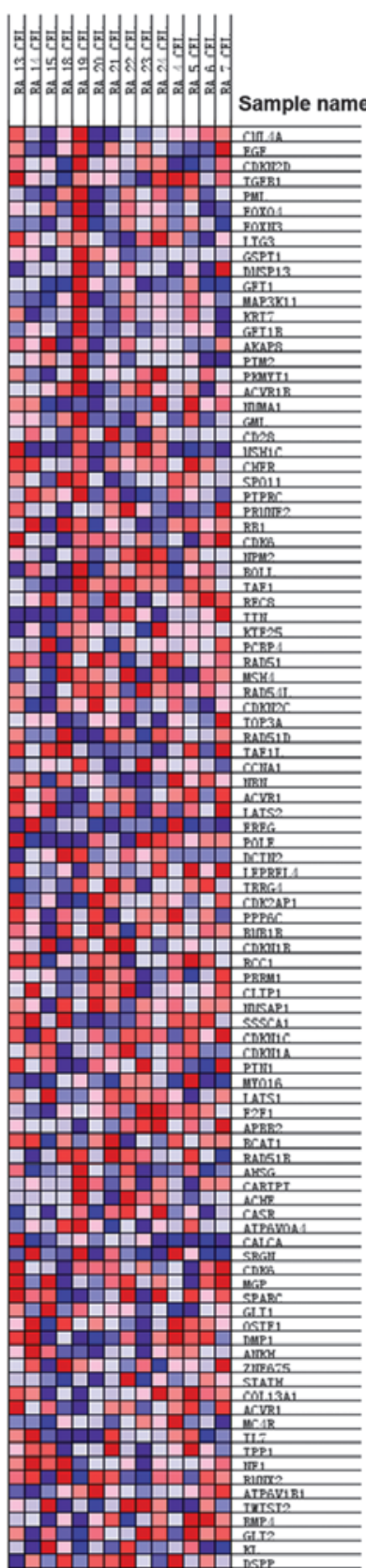

Figure 2. FGFR1 affects the cell cycle in osteosarcoma. (A) Kyoto Encyclopedia of Genes and Genomes analysis revealed that the (a) cell cycle was significantly affected in osteosarcoma ( $\mathrm{P}=0.003$ ), whereas no significant change was observed in the (b) bone remodeling process $(\mathrm{P}=0.369)$. (B) Heatmap analysis revealed increases in the experession of a broad spectrum of genes associated with the cell cycle, whereas the expression of other genes decreased. (C and D) Multiple cell cycle-associated genes were upregulated, while other genes were downregulated. FGFR1, fibroblast growth factor receptor 1.

(Fig. 1B). The elevated protein and mRNA levels of FGFR1 in osteosarcoma suggested that FGFR1 may be involved in osteosarcomagenesis.

FGFR1 affects the cell cycle in osteosarcoma. To investigate the possible pathways, which contributed to the effects of FGFR in osteosarcoma, the Kyoto Encyclopedia of Genes and Genomes (KEGG) database (http://www.kegg.jp/kegg/pathway.html) was accessed (Fig. 2). Notably, cell cycle was significantly affected in osteosarcoma (Fig. 2A(a); P=0.003). Bone remodeling is a ubiquitous process in bone diseases, therefore, the bone remodeling process was also assessed as a control. As expected, bone remodeling was not significantly affected
(Fig. 2A(b); $\mathrm{P}=0.369)$. As shown in Fig. 2C and $\mathrm{D}$, the cell cycle was significantly affected in osteosarcoma, with the upregulation of multiple cell cycle-associated genes and the downregulation of other genes downegulated. Furthermore, the present study performed microarray analysis of osteosarcoma using MATLAB, and to identify the genes and pathways that are involved in disease development, heatmap analysis was performed. The heatmap analysis demonstrated that multiple genes associated with the cell cycle were elevated, whereas certain other genes were decreased in osteosarcoma (Fig. 2B). Taken together, the data from the KEGG and microarray analyses suggested that the cell cycle is significantly affected by FGFR1 in osteosarcoma. 
A

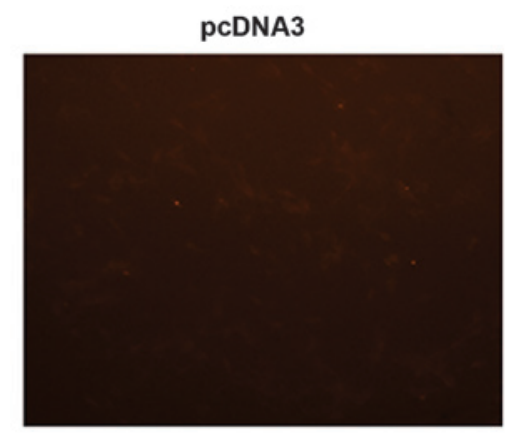

B

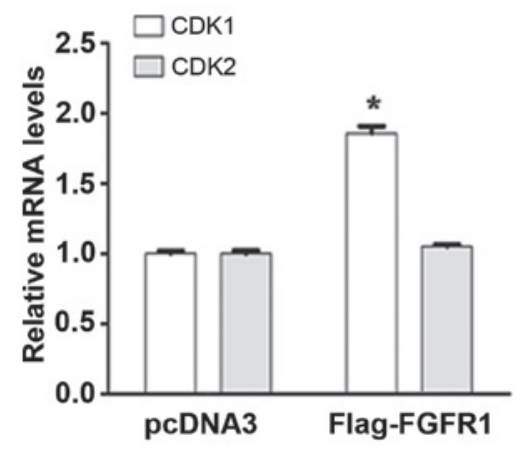

Flag-FGFR1

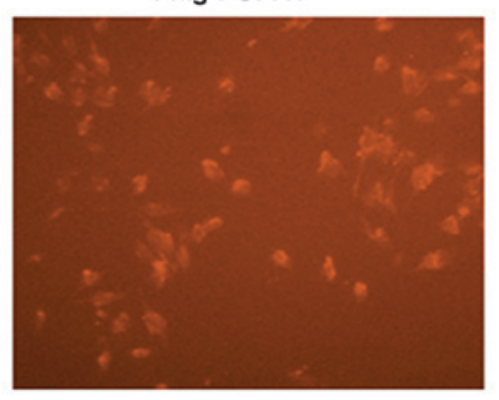

C

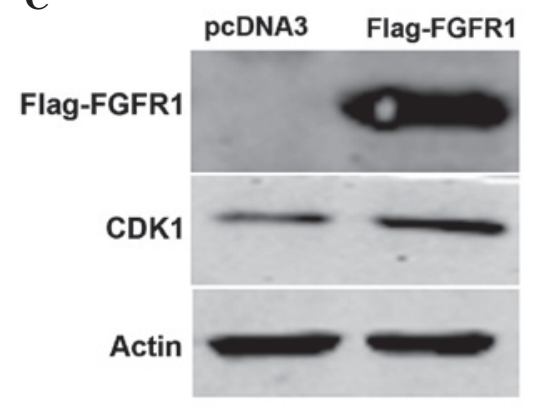

Figure 3. CDK1 is upregulated by FGFR1 in MG63 osteosarcoma cells. (A) Transfection efficiency was verified using a fluorescence microscope (magnification, x200). (B) Reverse transcription-quantitative polymerase chain reaction analysis of the mRNA levels of CDK1 and CDK2 in FGFR1-overexpressed MG63 cells. The mRNA expression of CDK1 was upregulated by FGFR1 overexpression ( $\mathrm{P}<0.05)$. Data are expressed as the mean \pm standard deviation. (C) Western blotting revealed that the protein expression of CDK1 increased following transfection of the MG63 cells with the FLAG-FGFR1 plasmid. CDK, cyclin-dependent kinase; FGFR1, fibroblast growth factor receptor 1.

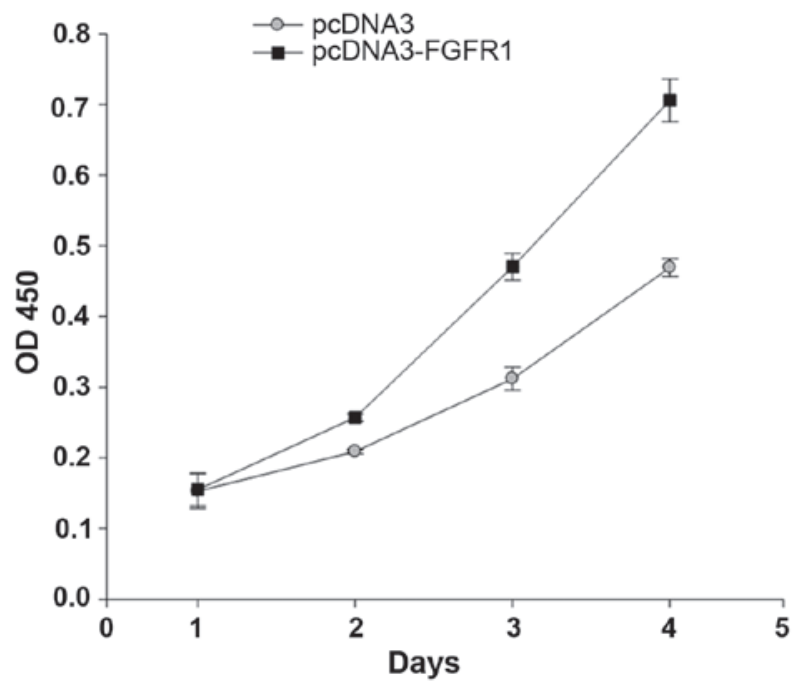

Figure 4. Overexpression of FGFR1 promotes MG63 cell proliferation. The MG63 cells were transfected with the FGFR1 expression plasmid. At $48 \mathrm{~h}$ post-transfection, the cells from FGFR1-overexpression group and control group were examined using a cell viability assay. The cells in the FGFR1-overexpressed group exhibited increased proliferative activity from day 2. On day 4, the number of cells in the FGFR1-overexpressed group were $\sim 1$-fold higher than those in the control group. FGFR1, fibroblast growth factor receptor 1 ; OD, optical density.

CDK1 is upregulated by FGFR1 in MG63 osteosarcoma cells. To further investigate the downstream molecules regulated by FGFR1, the MG63 cells were transfected with the Flag-FGFR1 expression plasmid. Transfection efficiency was visualized using a fluorescence microscope (Fig. 3A). Subsequently, total RNA was extracted and RT-qPCR analysis was performed to examine the expression of cell cycle molecules, including CDK1 and CDK2. Notably, the mRNA expression of CDK1 increased significantly in response to FGFR1 overexpression, whereas no significant change was observed in the mRNA level of CDK2 (Fig. 3B). Consistently, western blotting also confirmed that the protein level of CDK1 was upregulated following MG63 cell transfection with the pcDNA3-Flag-FGFR1 plasmid (Fig. 3C). These data suggested that FGFR1 targeted CDK1 to regulate the cell cycle in osteosarcoma.

Overexpression of FGFR1 promotes MG63 cell proliferation. To investigate the role of FGFR1 in osteosarcoma development, the MG63 cells were transfected with the pcDNA3-Flag-FGFR1 plasmid and a cell viability assay was performed. The absorbance at $450 \mathrm{~nm}$, which represents cell numbers was continuously determined for 5 days. The results demonstrated that the number of cells in the FGFR1-overexpressed group were higher than in the control group from day 2. Subsequently, the cell numbers increased markedly, with the FGFR1-overexpressed MG63 cells increasing $\sim 7$-fold by day 4 . Notably, the number of FGFR1-overexpressed MG63 cells increased by almost 1.7-fold, compared with the control group on day 4 (Fig. 4). These data lead to the conclusion that FGFR1 overexpression promoted MG63 cell growth. 


\section{Discussion}

Although substantial progress has been achieved through combination surgical resection and neoadjuvant chemotherapy in the treatment of human osteosarcoma, the survival rate of patients with osteosarcoma exhibiting localized diseases at diagnosis has remained at $\sim 70 \%$ since the mid-1980s, and the long-term survival rate of patients with metastatic or recurrent disease remains $<20 \%(32,33)$. Accordingly, increased understanding of the molecular mechanisms underlying osteosarcomagenesis, and the identification of novel molecular targets for osteosarcoma treatments, are urgently required. The present study investigated the role of FGFR1 in the development of human osteosarcoma, and examined the possible mechanisms underlying the involvement of FGFR1 in osteosarcoma.

Consistent with the expression profile in other types of cancer, the present study demonstrated that the expression level of FGFR1 was elevated in osteosarcoma tissues (Fig. 1). Overexpression of FGFR1 significantly promoted MG63 cell proliferation (Fig. 4), indicating that a high expression level of FGFR1 may be an indicator of osteosarcoma cell growth. Furthermore, the possible pathways that contribute to the role of FGFR1 in osteosarcoma were screened. KEGG database and heatmap analysis were common strategies that were used for high-throughput microarray analysis (34). With the assistance of these two tools, the present study demonstrated that cell cycle was significantly affected in the development of FGFR1-mediated osteosarcoma (Fig. 2A). Multiple gene expression levels were elevated, and others were decreased (Fig. 2B-D). Based on these findings, the present study upregulated the expression of FGFR in MG63 cells and, following verification of transfection efficiency (Fig. 3A), detected the expression of CDKs using RT-qPCR and western blot analysis. The results revealed that the expression of CDK1, but not CDK2, was significantly upregulated by FGFR1 in the MG63 cells (Fig. 3B and C). These findings indicated that FGFR1 may affect cell cycle by regulating the expression of CDK1.

CDKs are key regulators of the cell cycle and are under tight control by external and internal signals (35). The targeting of CDKs is always implicated in clinical anticancer drug developments $(11,36)$. The role of CDKs in neoplasia is also widely reported (11). In general, CDK1 and cyclin B1 are associated with the $\mathrm{G} 2 / \mathrm{M}$ phase; CDK2, cyclin $\mathrm{E}$ and cyclin A are associated with the G1/S transition and S phase; and CDK4 and cyclin D1 are associated with the G1 phase (37). In the present study, the upregulation of FGFR 1 caused a corresponding increase in the expression of CDK1, without affecting the level of CDK2 (Fig. 3B and C). These data suggested that FGFR1 may affect the G2/M phase. Of note, targeting CDK1, but not CDK4/6 or CDK2, is selectively lethal to MYC-dependent human breast cancer cells (38). Melatonin also selectively targets CDK1, but not CDK2, to promote breast cancer cell proliferation (37). These reports confirm that the promoting effect of FGFR1 may be due to the upregulation of CDK1, which is associated with the G2/M phase. However, the detailed mechanisms of how FGFR1 regulates CDK1 and affects the G2/M phase remain to be elucidated. As kinase activity is critical for FGFR1 and CDK function, the present study hypothesized that phosphorylation may be pivotal in
FGFR1-mediated cell cycle regulation. However, further investigation is required.

The present study provided evidence that FGFR1 promoted osteosarcoma cell proliferation, and that CDK1 may be critical in FGFR1-mediated osteosarcoma development. These findings may improve current understanding of molecule mechanisms underlying the development of osteosarcoma, and may provide novel insights into the molecular targeted therapy for osteosarcoma. The development of novel drugs targeting FGFR1 may be promising for osteosarcoma treatment and prevention.

\section{References}

1. Longhi A, Errani C, De Paolis M, Mercuri M and Bacci G: Primary bone osteosarcoma in the pediatric age: State of the art. Cancer Treat Rev 32: 423-436, 2006.

2. Kang K, Lee JH and Kim HG: Contralateral referred pain in a patient with intramedullary spinal cord metastasis from extraskeletal small cell osteosarcoma. J Spinal Cord Med 36: 695-699, 2013.

3. Chou AJ, Geller DS and Gorlick R: Therapy for osteosarcoma: Where do we go from here? Paediatr Drugs 10: 315-327, 2008.

4. Ahmad I, Iwata T and Leung HY: Mechanisms of FGFR-mediated carcinogenesis. Biochim Biophys Acta 1823: 850-860, 2012.

5. Liang G, Liu Z, Wu J, Cai Y and Li X: Anticancer molecules targeting fibroblast growth factor receptors. Trends Pharmacol Sci 33: 531-541, 2012.

6. Wesche J, Haglund K and Haugsten EM: Fibroblast growth factors and their receptors in cancer. Biochem J 437: 199-213, 2011.

7. Johnson DE and Williams LT: Structural and functional diversity in the FGF receptor multigene family. Adv Cancer Res 60: 1-41, 1993.

8. Chin K, DeVries S, Fridlyand J, Spellman PT, Roydasgupta R, Kuo WL, Lapuk A, Neve RM, Qian Z, Ryder T, et al: Genomic and transcriptional aberrations linked to breast cancer pathophysiologies. Cancer Cell 10: 529-541, 2006.

9. Gelsi-Boyer V, Orsetti B, Cervera N, Finetti P, Sircoulomb F Rougé C, Lasorsa L, Letessier A, Ginestier C, Monville F, et al: Comprehensive profiling of 8p11-12 amplification in breast cancer. Mol Cancer Res 3: 655-667, 2005.

10. Jacquemier J, Adelaide J, Parc P, Penault-Llorca F, Planche J, deLapeyriere $\mathrm{O}$ and Birnbaum D: Expression of the FGFR1 gene in human breast-carcinoma cells. Int J Cancer 59: 373-378, 1994.

11. McDonald ER III and El-Deiry WS: Cell cycle control as a basis for cancer drug development (Review). Int J Oncol 16: 871-886, 2000.

12. Roumiantsev S, Krause DS, Neumann CA, Dimitri CA, Asiedu F, Cross NC and Van Etten RA: Distinct stem cell myeloproliferative/T lymphoma syndromes induced by ZNF198-FGFR1 and BCR-FGFR1 fusion genes from 8p11 translocations. Cancer Cell 5: 287-298, 2004.

13. Xiao S, Nalabolu SR, Aster JC, Ma J, Abruzzo L, Jaffe ES Stone R, Weissman SM, Hudson TJ and Fletcher JA: FGFR1 is fused with a novel zinc-finger gene, ZNF198, in the $\mathrm{t}(8 ; 13)$ leukaemia/lymphoma syndrome. Nat Genet 18: 84-87, 1998.

14. Yagasaki F, Wakao D, Yokoyama Y, Uchida Y, Murohashi I, Kayano H, Taniwaki M, Matsuda A and Bessho M: Fusion of ETV6 to fibroblast growth factor receptor 3 in peripheral T-cell lymphoma with a $\mathrm{t}(4 ; 12)$ (p16;p13) chromosomal translocation. Cancer Res 61: 8371-8374, 2001.

15. Clark JC, Tichelaar JW, Wert SE, Itoh N, Perl AK, Stahlman MT and Whitsett J: FGF-10 disrupts lung morphogenesis and causes pulmonary adenomas in vivo. Am J Physiol Lung Cell Mol Physiol 280: L705-L715, 2001.

16. Marek L, Ware KE, Fritzsche A, Hercule P, Helton WR, Smith JE, McDermott LA, Coldren CD, Nemenoff RA, Merrick DT, et al: Fibroblast growth factor (FGF) and FGF receptor-mediated autocrine signaling in non-small-cell lung cancer cells. Mol Pharmacol 75: 196-207, 2009.

17. van Rhijn BW, van Tilborg AA, Lurkin I, Bonaventure J, de Vries A, Thiery JP, van der Kwast TH, Zwarthoff EC and Radvanyi F: Novel fibroblast growth factor receptor 3 (FGFR3) mutations in bladder cancer previously identified in non-lethal skeletal disorders. Eur J Hum Genet 10: 819-824, 2002. 
18. Greenman C, Stephens P, Smith R, Dalgliesh GL, Hunter C, Bignell G, Davies H, Teague J, Butler A, Stevens C, et al: Patterns of somatic mutation in human cancer genomes. Nature 446: $153-158,2007$.

19. Naski MC, Wang Q, Xu J and Ornitz DM: Graded activation of fibroblast growth factor receptor 3 by mutations causing achondroplasia and thanatophoric dysplasia. Nat Genet 13: 233-237, 1996.

20. Gru AA and Allred DC: FGFR1 amplification and the progression of non-invasive to invasive breast cancer. Breast Cancer Res 14: $116,2012$.

21. Shiang CY, Qi Y, Wang B, Lazar V, Wang J, Fraser Symmans W, Hortobagyi GN, Andre F and Pusztai L: Amplification of fibroblast growth factor receptor-1 in breast cancer and the effects of brivanib alaninate. Breast Cancer Res Treat 123 . 747-755, 2010.

22. Dutt A, Ramos AH, Hammerman PS, Mermel C, Cho J Sharifnia T, Chande A, Tanaka KE, Stransky N, Greulich H, et al: Inhibitor-sensitive FGFR1 amplification in human non-small cell lung cancer. PLoS One 6: e20351, 2011.

23. Tran TN, Selinger CI, Kohonen-Corish MR, McCaughan BC, Kennedy CW, O'Toole SA and Cooper WA: Fibroblast growth factor receptor 1 (FGFR1) copy number is an independent prognostic factor in non-small cell lung cancer. Lung Cancer 81: 462-467, 2013

24. Ren M, Hong M, Liu G, Wang H, Patel V, Biddinger P, Silva J, Cowell J and Hao Z: Novel FGFR inhibitor ponatinib suppresses the growth of non-small cell lung cancer cells overexpressing FGFR1. Oncol Rep 29: 2181-2190, 2013.

25. Acevedo VD, Gangula RD, Freeman KW, Li R, Zhang Y, Wang F, Ayala GE, Peterson LE, Ittmann M and Spencer DM: Inducible FGFR-1 activation leads to irreversible prostate adenocarcinoma and an epithelial- to -mesenchymal transition. Cancer Cell 12 559-571, 2007.

26. Lehnen NC, von Mässenhausen A, Kalthoff H, Zhou H, Glowka T, Schütte U, Höller T, Riesner K, Boehm D, Merkelbach-Bruse S, et al: Fibroblast growth factor receptor 1 gene amplification in pancreatic ductal adenocarcinoma. Histopathology 63: 157-166, 2013

27. Freier K, Schwaenen C, Sticht C, Flechtenmacher C, Mühling J, Hofele C, Radlwimmer B, Lichter P and Joos S: Recurrent FGFR1 amplification and high FGFR1 protein expression in ora squamous cell carcinoma (OSCC). Oral Oncol 43: 60-66, 2007.
28. Simon R, Richter J, Wagner U, Fijan A, Bruderer J, Schmid U, Ackermann D, Maurer R, Alund G, Knönagel H, et al: High-throughput tissue microarray analysis of 3p25 (RAF1) and 8p12 (FGFR1) copy number alterations in urinary bladder cancer. Cancer Res 61: 4514-4519, 2001.

29. Gorringe KL, Jacobs S, Thompson ER, Sridhar A, Qiu W, Choong DY and Campbell IG: High-resolution single nucleotide polymorphism array analysis of epithelial ovarian cancer reveals numerous microdeletions and amplifications. Clin Cancer Res 13: 4731-4739, 2007.

30. Missiaglia E, Selfe J, Hamdi M, Williamson D, Schaaf G, Fang C, Koster J, Summersgill B, Messahel B, Versteeg R, et al: Genomic imbalances in rhabdomyosarcoma cell lines affect expression of genes frequently altered in primary tumors: An approach to identify candidate genes involved in tumor development. Genes Chromosomes Cancer 48: 455-467, 2009.

31. Guimarães JM, Guimarães IC, Duarte ME, Vieira T, Vianna VF, Fernandes MB, Vieira AR and Casado PL: Polymorphisms in BMP4 and FGFR1 genes are associated with fracture non-union. J Orthop Res 31: 1971-1979, 2013.

32. Chou AJ, Merola PR, Wexler LH, Gorlick RG, Vyas YM, Healey JH, LaQuaglia MP, Huvos AG and Meyers PA: Treatment of osteosarcoma at first recurrence after contemporary therapy: The memorial sloan-kettering cancer center experience. Cancer 104: 2214-2221, 2005.

33. He H, Ni J and Huang J: Molecular mechanisms of chemoresistance in osteosarcoma (Review). Oncol Lett 7: 1352-1362, 2014.

34. Mougeot JL, Li Z, Price AE, Wright FA and Brooks BR: Microarray analysis of peripheral blood lymphocytes form ALS patients and the SAFE detection of the KEGG ALS pathway. BMC Med Genomics 25: 74-80, 2011.

35. Morgan DO: Principles of CDK regulation. Nature 374: 131-134, 1995.

36. Benson C, Kaye S, Workman P, Garrett M, Walton M and de Bono J: Clinical anticancer drug development: Targeting the cyclin-dependent kinases. Br J Cancer 92: 7-12, 2005.

37. Liu L, Xu Y and Reiter RJ: Melatonin inhibits the proliferation of human osteosarcoma cell line MG-63. Bone 55: 432-438, 2013.

38. Kang J, Sergio CM, Sutherland RL and Musgrove EA: Targeting cyclin-dependent kinase 1 (CDK1) but not CDK4/6 or CDK2 is selectively lethal to MYC-dependent human breast cancer cells. BMC Cancer 14: 32, 2014. 